\title{
OS SENTIDOS DO TRABALHO PARA DOCENTES DE PÓS-GRADUAÇÃO STRICTO SENSU: UM ESTUDO COM DOCENTES DAS ÁREAS DE ADMINISTRAÇÃO E EDUCAÇÃO
}

\author{
THE WORK MEANINGS OF THE GRADUATE STRICTO SENSU PROFESSORS: A STUDY WITH \\ PROFESSORS IN THE AREAS OF BUSINESS ADMINISTRATION AND EDUCATION
}

\section{LOS SENTIDOS DEL TRABAJO PARA DOCENTES DE POSGRADO STRICTO SENSU: UN ESTUDIO CON DOCENTES DE LAS ÁREAS DE ADMINISTRACIÓN Y EDUCACIÓN}

\author{
MALENA PILONI PETRI \\ Mestre \\ IMED Faculdade Meridional - Brasil \\ malena.pp@hotmail.com \\ ORCID: http://orcid.org/0000-0002-7850-6496
}

SHALIMAR GALLON

Doutora

IMED Faculdade Meridional - Brasil shalimar.canfield@imed.edu.br

ORCID: http://orcid.org/0000-0002-8830-4433

\section{ELEM DUARTE VAZ}

Mestranda

IMED Faculdade Meridional - Brasil elemduartevaz@gmail.com

ORCID: http://orcid.org/0000-0002-9584-4497

Submetido em: 16/04/2018

Aprovado em: 24/01/2019

Doi: alcance.v25n3(Set/Dez).p366-380

\begin{abstract}
RESUMO
O objetivo da pesquisa é analisar os sentidos do trabalho para docentes de pós-graduação stricto sensu das áreas de Administração e Educação do Estado do Rio Grande do Sul. A pesquisa realizada é de natureza descritiva com abordagem qualitativa. A coleta de informações foi realizada por meio de 27 entrevistas com roteiro semiestruturado e as informações analisadas com base na técnica de análise de conteúdo. Para os entrevistados, um trabalho que tem sentido proporciona satisfação pessoal, realização e identidade, está organizado de forma que apresente objetivos claros e resultados úteis, que proporcione possibilidades de estabelecer relações interpessoais, inserção social e que seja moralmente aceitável. Os resultados mesclam os sentidos do trabalho encontrados por Morin (2001), Morin, Tonelli e Pliopas (2003), Oliveira, Piccinini, Fontoura e Schuweig (2004) e Piccinini, Oliveira, Fontoura e Schweig (2005), apresentando as características de um trabalho que tem sentido. Gerencialmente, os resultados alcançados podem auxiliar as IES em questões referentes à organização do trabalho docente, como a burocracia, os excessos de reuniões, a carga excessiva de trabalho e a má divisão dos cargos de gestão.
\end{abstract}

Palavras-chave: Sentido do Trabalho. Trabalho Docente. Pós-Graduação. 


\section{ABSTRACT}

The objective of the research is to analyze the work meanings of the graduate stricto sensu professors of the areas of Administration and Education of the Rio Grande do Sul State. The research carried out is of a descriptive nature with a qualitative approach. The data collection was conducted through a 27 semi-structured interview script and the information analyzed based on the technique of content analysis. For those interviewed, meaningful work provides personal satisfaction, achievement, and identity. It is a work that is organized to present clear goals and useful results, which provides possibilities for establishing interpersonal relationships, social insertion and that is morally acceptable. In this way, the results combine the meanings of the work found by Morin (2001) and Morin, Tonelli and Pliopas (2003), to those reported by Oliveira, Piccinini, Fontoura and Schuweig (2004) and Piccinini, Oliveira, Fontoura and Schweig (2005) in his studies, showing the characteristics of a job that makes sense. Managerially the results can help the HEls in questions related to the organization of teaching work, such as bureaucracy, excess meetings, excessive workload, and poor division of management positions.

Keywords: Work meaning. Professor. Postgraduate.

\section{RESUMEN}

El objetivo de la investigación es analizar los sentidos del trabajo para docentes de posgrado stricto sensu de las áreas de Administración y Educación del Estado de Rio Grande do Sul. La investigación realizada es de naturaleza descriptiva con abordaje cualitativo. La recolección de informaciones fue realizada por medio de 27 entrevistas con guion semiestructurado y las informaciones analizadas con base en la técnica de análisis de contenido. Para los entrevistados, un trabajo que tiene sentido proporciona satisfacción personal, realización e identidad. Es un trabajo que está organizado de forma que presente objetivos claros y resultados útiles, que proporcione posibilidades de establecer relaciones interpersonales, inserción social y que sea moralmente aceptable. De esta forma, estos resultados mezclan los sentidos del trabajo encontrados por Morin (2001) y Morin, Tonelli y Pliopas (2003), a los relatados por Oliveira, Piccinini, Fontoura y Schuweig (2004) y Piccinini, Oliveira, Fontoura y Schweig (2005) en sus estudios, presentando las características de un trabajo que tiene sentido. En general, los resultados alcanzados pueden ayudar a las IES en cuestiones referentes a la organización del trabajo docente, como la burocracia, excesos de reuniones, carga excesiva de trabajo, y mala división de los cargos de gestión.

Palabras clave: Sentido del trabajo. Trabajo Docente. Posgrado.

\section{INTRODUÇÃO}

A sociedade contemporânea tem passado por transformações, resultando em alterações nas estruturas e nas formas de trabalho, provenientes dos impactos da globalização, reestruturação produtiva, mudanças contratuais, competitividade, flexibilização e precarização (Tolfo, Coutinho, Almeida, Nbaasch, \& Cugnier, 2005; Tolfo \& Piccinini, 2007; Prates, Silva, \& Piccinini, 2014). As percepções de que o trabalho possui sentido é um tema de crescente relevância para pesquisadores, gerentes e organizações que procuram aumentar a satisfação com o trabalho e, consequentemente, aumentar também as contribuições para sociedade e organizações (Steger, Dik, \& Duffy, 2012). Assim, o trabalho tem sido investigado por diversas áreas do conhecimento em função da sua importância e pelas suas constantes transformações, buscando compreender os impactos dessas transformações nas organizações para o trabalhador e para a sociedade (Tolfo \& Piccinini, 2007; Prates et al., 2014).

Neste cenário, apesar das transformações que ocorreram no mundo do trabalho, este continua sendo central na vida dos sujeitos. É por meio do trabalho que o sujeito transforma a natureza e a si mesmo, a partir dos sentidos atribuídos às coisas que ele conquista e o quanto de si ele consegue colocar naquilo que faz. Dessa forma, é importante que o trabalho tenha sentido para quem o realiza, pois vai além de trabalhar em troca de remuneração, tornando o indivíduo parte de um grupo, constrói redes de relações sociais (Morin, 2001, 2002), permite a sobrevivência, a variedade das tarefas, aprendizagem, reconhecimento, segurança e autonomia (Morin, Tonelli, \& Pliopas, 2003, 2007). Assim, as questões relativas ao sentido do trabalho tornaram-se objeto de crescente interesse (Tolfo et al., 2005).

Uma das profissões que tem se apresentado como foco de pesquisa é a de docentes, principalmente daqueles que escolhem a carreira acadêmica em função de englobar diversas atividades. A carga de trabalho dos docentes é cada vez maior, pois eles têm assumido mais atividades e funções, o que em conjunto com as avaliações da CAPES, que privilegiam o produtivismo, torna a carreira docente uma das mais estressantes 
(Freitas, 2007b) em função da sobreposição de exigências, como aulas, relatórios, eventos, editais, palestras, busca por financiamento, supervisões, avaliação e produção de artigos para periódicos e eventos, avaliação da CAPES, escrita, leituras, reuniões e orientações.

Nesse contexto, diversos autores discutem a precarização dos Programas de Pós-Graduação (PPG) stricto sensu, o acúmulo de funções dos docentes e o sistema de avaliação CAPES, como também sua contribuição para 0 aumento da carga de trabalho docente. É valorizada a quantidade produzida em detrimento da qualidade, 0 que enfraquece a formação de mestres e doutores e causa adoecimento dos professores (Freitas, 2007a; Moreira \& Velho, 2008; Bianchetti \& Machado, 2009; Oliveira \& Freitas, 2009; Alcadipani, 2011; Maués \& Mota Jr., 2011; Patrus \& Lima, 2014; Pizzio \& Klein, 2015; Vosgerau, Orlando, \& Meyer, 2016).

Em meio à precarização do trabalho e às mudanças na carreira acadêmica, ainda existem gratificações, como o papel que o docente assume na vida de seus alunos e orientandos, sua identificação com os pares, a liberdade de se expressar e de escolher os temas de pesquisa (Freitas, 2011). É um trabalho que possibilita inserção social, o estabelecimento de relacionamento interpessoal, o reconhecimento, as oportunidades de aprendizado, a autonomia e a flexibilidade de horários (Nascimento, Costa, Salvá, \& Moura, 2016). Sendo assim, quando os sujeitos compreendem a importância do seu trabalho e o impacto que suas tarefas têm sobre os outros, eles percebem mais sentido no trabalho e isso pode até mesmo elevar a motivação e o desempenho (Berg, Dutton, \& Wrzesniewski, 2013).

Para compreender o tema proposto, foi realizada uma busca por artigos publicados nos últimos dez anos nas bases de dados dos periódicos nacionais e internacionais. O levantamento bibliográfico foi realizado em junho de 2017 por meio de pesquisa nos sites de periódicos nacionais da área de Administração Pública e Administração de Empresas, Ciências Contábeis e Turismo, com Qualis A1 até B2 na classificação de 2015. Os termos pesquisados foram: docente, sentido do trabalho, sentido do trabalho docente, professor, stricto sensu, pósgraduação, docente universitário, professor universitário e carreira acadêmica. A pesquisa envolveu 248 periódicos da área e, após esta etapa, foi realizada uma busca nas bases Academic Search Premier (EBSCO), Scielo e Spell Scientific Periodicals Eletronic Library utilizando os mesmos termos.

Como resultado da pesquisa bibliográfica foram encontrados 668 artigos. Após a leitura dos resumos, encontraram-se 47 artigos que tratavam de alguma forma do trabalho docente na PPG stricto sensu. Os temas mais abordados foram a intensificação do trabalho, a pressão por publicações, a avaliação da CAPES e da instituição, com 18 publicações. Também foram encontradas 14 publicações relativas à formação para a docência e práticas de ensino e pesquisa na PPG stricto sensu. As competências gerenciais de docência e desempenho foram encontradas em cinco trabalhos, enquanto que o trabalho docente, sua agenda, rotina e relações de trabalho foram abordados em quatro artigos. Já a relação orientador-orientando foi abordada em três trabalhos e as redes de colaboração e coautorias em três artigos.

Em relação ao sentido atribuído pelos docentes ao trabalho, foram encontrados cinco artigos, porém todos foram realizados com docentes de graduação. São artigos que relacionam sentido do trabalho, qualidade de vida e orientação para o trabalho (Vilas Boas \& Morin, 2015, 2016, 2017), ou que estudam o sentido do trabalho para docentes idosos (Nascimento et al., 2016), e o sentido do trabalho, utilizando como base teórica a psicodinâmica do trabalho (Coutinho, Magro, \& Budde, 2011).

Dessa forma, tem-se a seguinte questão de pesquisa: qual o sentido do trabalho para docentes de PPG stricto sensu das áreas de Administração e Educação? A fim de responder essa pergunta, esta pesquisa busca analisar os sentidos do trabalho para docentes de PPG stricto sensu da área de Administração e da Educação.

A escolha dessas áreas se deu no intuito de agregar diferentes perspectivas à pesquisa, em função das suas especificidades, sendo a Administração voltada para a análise crítica de diferentes teorias e práticas de gestão, com estudos objetivando a geração de conhecimento relacionado à gestão das organizações e sua relação com o desenvolvimento da sociedade. Os professores de PPG stricto sensu da área de Administração, além da boa didática e experiência acadêmica, devem possuir experiência profissional, não acadêmica, sendo este um atributo de grande importância atribuído pelos alunos (Costa, 2006). Dentre as atividades do professor na área de Administração, se encontram a tomada de decisão, o planejamento, a liderança e o controle, que estão voltados para a gestão organizacional, sejam eles humanos, de materiais, financeiros, mas que têm por objetivo alcançar os propósitos organizacionais de maneira eficiente e efetiva.

Já a área da Educação é voltada à busca da qualificação de professores e educadores, com foco nas práticas pedagógicas, investigando a origem, a organização, a natureza e a finalidade do trabalho pedagógico por

Revista Alcance - Eletrônica - vol. 25 - n. 3 - Set./Dez. 2018 
meio de seus fundamentos constitutivos, processo pedagógico e políticas educacionais. Os programas de PPG stricto sensu da área de educação possuem como demanda a formação de professores da educação de diversas áreas (Duarte, 2006), o que pode carecer de uma dinâmica mais heterogênea dos professores que ministram nesta área.

Além disso, o professor de PPG stricto sensu da área de educação, ao mesmo tempo que cumpre o processo para sua formação para atingir a titulação de mestre e/ou docente - o que valoriza sua carreira -, também encontra possibilidade de cumprir as exigências e as necessidades de conhecimentos ligados à sua condição de docente (Duarte, 2006). 0 professor da área de Educação ainda difere do professor da Administração, pois deve planejar, organizar e ponderar as demandas coletivas de forma mais democrática, além de refletir sobre as questões mais humanas que envolvem todos os que atuam na realidade docente, priorizando, assim, 0 desenvolvimento do aluno. Frente a essas características, o trabalho pode se apresentar diferentemente para os docentes de cada área, o que justifica o interesse de analisar as áreas em questão.

\section{SENTIDO DO TRABALHO E A DOCÊNCIA}

$O$ trabalho é uma atividade complexa, polissêmica, multifacetada, que exige diferentes olhares para sua compreensão (Neves, Nascimento, Felix Jr, Da Silva, \& De Andrade, 2017). Ele também desempenha papel poderoso na forma de como as pessoas entendem suas vidas, como elas preenchem seus lugares e como entendem o mundo ao seu redor (Steger \& Dik, 2009). Por ser uma atividade central que estrutura a vida dos indivíduos, o trabalho ocupa um papel importante na sociedade (Tolfo et al., 2005), sendo considerado crucial para sobrevivência da humanidade (Drenth, 1991). A associação entre o trabalho e os benefícios que ele traz, tanto para os trabalhadores quanto para as organizações, é umas das razões que reforça a importância de se estudar 0 sentido do trabalho (Steger et al., 2012).

Sendo assim, pesquisas sobre o tema concentraram-se em questões em como os empregados encontram sentido em seu trabalho, como alguns trabalhos similares possuem sentidos diferentes para os trabalhadores e como o sentido do trabalho muda ao longo do tempo, implicando, assim, em crenças diferentes sobre o sentido do trabalho (Rosso, Dekas, \& Wrzesniewski, 2010). Além disso, diversas mudanças no contexto social, como o aumento dos níveis educacionais, a inserção de mulheres, imigrantes e deficientes no mercado de trabalho, também podem influenciar as concepções sobre o direito de trabalhar e consequentemente impactam no sentido do trabalho (Drenth, 1991).

Portanto, seu conceito vem passando por múltiplas transformações produtivas e tecnológicas, emergindo, assim, estudos sobre o desemprego, a alienação, a precarização e a reestruturação produtiva (Neves et al., 2017; Tolfo et al., 2005). Em meio a essas transformações, os estudos sobre o sentido do trabalho vêm sendo realizados em diferentes áreas do conhecimento (Rosso et al., 2010), reforçando seu caráter multidisciplinar e passível de múltiplas perspectivas teóricas (Tolfo \& Piccinini, 2007).

Dentre as várias perspectivas que tratam do sentido do trabalho, encontra-se a do sociólogo Antunes (2009), propondo que, para que se tenha uma vida dotada de sentido, supõe-se que a vida dentro do trabalho seja também dotada de sentido, sendo incompatível uma vida cheia de sentido com uma vida sem sentido no trabalho. Já a compreensão de sentido do trabalho de Dejours (2013) está relacionada com a construção da identidade, a satisfação pessoal e o prazer, integrando o indivíduo ao todo social (Dejours, 2013; Heloani \& Lancman, 2004).

Apesar de existirem outras perspectivas, no presente estudo será adotada a perspectiva de sentido do trabalho de Morin $(2001,2002)$ e Morin et al. $(2003,2007)$. Segundo as autoras, um trabalho que tem sentido é realizado de forma eficiente, gerando um resultado útil; é satisfatório em sua realização, permitindo que o sujeito seja criativo, desenvolva suas competências, seja responsável e tenha autonomia; é um trabalho moralmente aceito, que contribui para a sociedade e permite inserção social. É um trabalho que é fonte de boas experiências e relações humanas; garante segurança e possibilita autonomia, com boas condições de trabalho, proporcionando um salário justo e possibilidade de melhora na qualidade de vida; e mantém as pessoas ocupadas, com rotinas e carga de trabalho adequadas.

Sendo assim, a partir da perspectiva de sentido do trabalho de Morin $(2001,2002)$ e Morin et al., (2003, 2007), os sentidos do trabalho foram classificados em três dimensões: individual, organizacional e social. $\mathrm{Na}$ dimensão individual, um trabalho que tem sentido é fonte de prazer, orgulho, identificação, criatividade, responsabilidade, autonomia, oportunidades de crescimento e aprendizado. $\mathrm{Na}$ dimensão organizacional, é atribuído sentido ao trabalho devido a sua utilidade, seu propósito, conhecimento do processo produtivo, 
reconhecimento por membros da organização e pela oportunidade de se relacionar com outras pessoas. Já a dimensão social é descrita como uma maneira de pertencer ao conjunto social e contribuir com a sociedade, transcendendo as questões individuais e organizacionais (Morin et al., 2003, 2007).

Neste cenário, o sentido do trabalho tornou-se objeto de estudos de forma mais intensa a partir da década de 1970 (Tolfo et al., 2005). Os primeiros estudos empíricos acerca da temática dos sentidos do trabalho foram desenvolvidos por Hackman e Oldham (1975), com foco no diagnóstico e no replanejamento do trabalho e como objetivo de melhorar a motivação e a produtividade dos empregados. Para os autores, um trabalho que possui sentido deve ser importante, útil e legítimo para o trabalhador que o executa, apresentando três características fundamentais: a identificação do trabalhador com uma variedade de tarefas que o possibilita utilizar suas competências; oferecer possibilidade de realizar algo com começo e fim demonstrando resultados; e oferecer retorno ao sujeito sobre seu desempenho (Hackman \& Oldham, 1975).

Já no final da década de 1980, um grupo de pesquisadores internacional conhecido como Meaning of Work (MOW) realizou uma pesquisa comparativa acerca das diferentes definições de trabalho em oito países (Neves et al., 2017; Bastos, Pinho, \& Costa, 1995; Morin, 2001; Bendassolli, 2009). Os autores consideraram o significado do trabalho como um construto multidimensional, constituído pelo contexto organizacional, pessoal, ambiental, no qual o indivíduo está inserido (Neves et al., 2017; Rigon \& Vilela, 2004; Tolfo \& Piccinini, 2007; Bendassolli, 2009). Os dados do estudo foram estruturados em doze fatores e agrupados em três dimensões finais: centralidade do trabalho; normas sociais sobre o trabalho; e resultados ou produtos valorizados do trabalho (Rogon \& Vilela, 2004). Diante dessas três dimensões, o sentido do trabalho é entendido como um elemento da realidade social que se constrói e se reproduz, interage com diferentes variáveis pessoais e sociais e influencia as ações das pessoas (MOW, 1987).

Dada a sua importância, estudos sobre o sentido do trabalho também foram realizados com professores. Vilas Boas e Morin (2016) avaliaram fatores de qualidade de vida de 274 professores do Brasil e de 252 professores do Canadá. Os resultados apontaram que os professores canadenses possuem maior autonomia, reconhecimento no seu trabalho e oportunidades de desenvolvimento profissional que os professores brasileiros. Já a carga mental e as horas semanais trabalhadas são maiores para os professores brasileiros. Dessa forma, os fatores relacionados às características e às relações de trabalho se relacionam positivamente com o sentido do trabalho e, ao contrário, as cargas de trabalho física e mental se relacionam negativamente com o sentido do trabalho (Vilas Boas \& Morin, 2016).

Vários outros estudos abordaram a temática sentido do trabalho para professores como: sofrimento e adoecimento de docentes (Forattini \& Lucena, 2015); sentido do trabalho docente e suas competências (Karawejczyk \& Estivalete, 2002); vivências de prazer e sofrimento dos docentes (Menezes, Nepomuceno, \& Batista-dos-Santos, 2011); sentido do trabalho relacionado à autonomia, à autodeterminação e à liberdade dos docentes (Silva \& Braga, 2014), à qualidade de vida e à orientação para o trabalho (Vilas Boas \& Morin, 2015, 2016, 2017); o sentido do trabalho para docentes idosos (Nascimento et al., 2016); e o sentido do trabalho utilizando como base teórica a psicodinâmica do trabalho (Coutinho et al., 2011). Porém, os estudos aqui citados foram realizados com docentes universitários de cursos de graduação. As buscas realizadas à procura de trabalhos sobre os sentidos atribuídos ao trabalho por professores de PPG stricto sensu não obtiveram resultados, o que salienta a importância da temática e do grupo de profissionais a ser estudado.

\section{PROCEDIMENTOS METODOLÓGICOS}

Para atingir os objetivos da presente pesquisa, foi realizado um estudo descritivo de caráter qualitativo. A coleta das informações foi realizada por meio de entrevistas com roteiro semiestruturado, por possibilitarem a inclusão de outras perguntas, sua reordenação e também interações, caso fossem necessárias, tendo em vista 0 alcance dos objetivos da pesquisa (Godoi \& Mattos, 2010). O instrumento de coleta utilizado foi elaborado com base na literatura estudada (Figura 1) e passou por um estudo-piloto com seis docentes da IES Priv 1. 0 estudopiloto permitiu ao pesquisador certificar-se quais, dentre as questões abordadas, estavam claras, eram relevantes e que faziam sentido na realidade social dos entrevistados (Godoy, 2005). Após a análise das informações obtidas, foram realizadas as alterações necessárias no roteiro de entrevistas, sendo eliminadas as questões que resultaram em informações repetidas. 
O roteiro foi dividido em quatro blocos, os quais abordam o perfil dos entrevistados, questões referentes ao trabalho, à descrição das atividades do trabalho dos docentes e às percepções dos entrevistados sobre 0 sentido do trabalho.

\begin{tabular}{|l|l|}
\hline Categorias & Autores base \\
\hline $\begin{array}{l}\text { Dimensão individual: satisfação pessoal, independência e } \\
\text { sobrevivência, realização, prazer, identidade, crescimento e } \\
\text { aprendizagem }\end{array}$ & $\begin{array}{l}\text { Morin et al. (2003), Oliveira, Piccinini, Fontoura e Schuweig } \\
\text { (2004) e Piccinini, Oliveira, Fontoura e Schuweig (2005) }\end{array}$ \\
\hline $\begin{array}{l}\text { Dimensão organizacional: utilidade, relacionamento } \\
\text { interpessoal, organização do trabalho }\end{array}$ & $\begin{array}{l}\text { Morin et al. (2003), Oliveira et al. (2004) e Piccinini et al. } \\
(2005)\end{array}$ \\
\hline Dimensão social: contribuição social, inserção social & $\begin{array}{l}\text { Morin et al. (2003), Oliveira et al. (2004) e Piccinini et al. } \\
(2005)\end{array}$ \\
\hline Organização do trabalho & $\begin{array}{l}\text { Morin (2001), Morin et al. (2003), Oliveira et al. (2004) e } \\
\text { Piccinini et al. (2005) }\end{array}$ \\
\hline Sentido do trabalho & $\begin{array}{l}\text { Morin (2001), Morin et al. (2003), Oliveira et al. (2004) e } \\
\text { Piccinini et al. (2005) }\end{array}$ \\
\hline
\end{tabular}

Figura 1: Roteiro de entrevista conforme as referências utilizadas em sua elaboração

Fonte: Elaborada pelas autoras (2018).

As entrevistas foram agendadas via e-mail e realizadas nas próprias instituições em que os docentes trabalhavam. A coleta foi realizada individualmente e as entrevistas gravadas mediante autorização dos participantes do estudo. Tanto as identidades dos participantes quanto das IES foram preservadas. As IES são identificadas como Priv 1, Publi e Priv 2 e os docentes como E1, E2, E3 e assim até o E27.

$\mathrm{Na}$ pesquisa qualitativa, a seleção de quem e quantas pessoas serão entrevistadas não segue a lógica de proporcionalidade e representatividade das técnicas quantitativas, o que atribui ao pesquisador a liberdade de ampliar a quantidade de participantes ou aprofundar a conversação, tendo como base 0 desenvolvimento do trabalho (Godoi \& Mattos, 2010). Nesse sentido, as entrevistas foram concluídas quando a saturação das informações coletadas foi atingida. Para tanto, quando as informações começaram a se repetir e novas informações deixaram de aparecer, ou seja, quando não foram encontrados dados adicionais que possibilitassem o acréscimo de propriedades a uma categoria, a coleta de informações foi encerrada (Flick, 2009; Godoi \& Mattos, 2010).

O grupo de entrevistados é composto por docentes de PPG stricto sensu em Administração (PPGA) e Educação (PPGEdu) de três IES reconhecidas pela CAPES, localizadas no estado do Rio Grande do Sul (RS). A escolha dessas áreas se deu no intuito de agregar diferentes perspectivas à pesquisa, em função das suas especificidades, conforme já exposto.

Apesar do estudo não ser comparativo, as IES tinham diferentes características para agregar diferentes percepções de docentes, independentemente da situação da IES. Assim, as IES foram escolhidas em função de acessibilidade aos entrevistados, por preencherem os requisitos da pesquisa (serem instituições estáveis e por possuírem PPG stricto sensu com cursos de mestrado acadêmico, mestrado profissional e doutorado nas áreas pesquisadas), bem como por apresentarem diferentes características, visto que a IES Priv 2 é uma IES privada, comunitária e confessional da região metropolitana do estado do RS; a Publi é uma IES pública da capital do estado do RS; e a IES Priv 1 é uma IES privada comunitária da Mesorregião do Noroeste do estado do RS.

Na IES Priv 1, o PPGEdu teve início em 1997 e, atualmente, possui conceito CAPES cinco, enquanto que o PPGA 1 iniciou suas atividades em 2015 e possui conceito CAPES três. Na IES Priv 2, o PPGEdu surgiu em 1994 e possui conceito CAPES sete. Já o PPGA teve início em 2000 e, atualmente, possui conceito CAPES seis. E, por fim, na IES Publi, o PPGEdu teve início em 1972 e possui conceito CAPES seis. O PPGA iniciou suas atividades em $1972 \mathrm{e}$, atualmente, possui conceito CAPES cinco.

O contato com os docentes teve início no dia 02 de setembro de 2017, com o envio dos primeiros convites por e-mail para participar da pesquisa. A primeira entrevista foi realizada no dia 12 de setembro de 2017 e 0 encerramento da coleta das informações ocorreu no dia 07 de dezembro de 2017 com a realização da última entrevista. Foram convidados para participar da pesquisa todos os docentes dos PPGA e PPGEdu das três IES selecionadas, somando assim 175 docentes convidados. No entanto, vinte e sete docentes (dezoito da área de Administração e nove da área de Educação) manifestaram vontade de participar da pesquisa e foram 
entrevistados. Em função da dificuldade em obter retorno dos docentes não foram feitas distinções em relação ao sexo, idade e tempo de vínculo institucional.

A idade dos entrevistados variou entre 32 e 75 anos. Foram entrevistadas 10 mulheres e 17 homens, sendo que, dentre todos os entrevistados, 21 eram casados. O tempo de trabalho na IES foi em média de 16,4 anos, variando entre 02 e 35 anos. 0 tempo de trabalho no PPG foi em média de 8,4 anos, variando entre 01 e 31 anos. As entrevistas totalizaram 21 horas e 29 minutos de conversação e 182 páginas de transcrições.

Para a análise e interpretação das informações, foi utilizada a técnica de análise de conteúdo, que é um conjunto de técnicas de análise que consiste na explicitação, na sistematização e na expressão das mensagens (Bardin, 2011). A análise partiu de três categorias intermediárias elencadas a priori com base na dimensão individual, organizacional e social apresentadas por Morin et al., $(2003,2007)$. As categorias iniciais foram surgindo conforme o desenvolvimento do trabalho de categorização e foram sendo organizadas de acordo com o referencial teórico utilizado, resultando em nove categorias (Figura 2).

Para auxiliar no processo de organização e categorização das informações, foi utilizado o software MaxQda versão 2007. 0 uso do software auxilia na organização das informações, facilita o processo e funciona como um apoio para a análise qualitativa, porém o mesmo não realiza a análise, sendo a compreensão do texto uma atividade humana, realizada pelos pesquisadores (Bandeira-de-Mello, 2010).

\begin{tabular}{|c|c|c|}
\hline Categorias Iniciais & $\begin{array}{l}\text { Categorias } \\
\text { Intermediárias }\end{array}$ & Categoria Final \\
\hline $\begin{array}{l}\text { Satisfação Pessoal - Realização pessoal, desafios a serem superados, } \\
\text { percepção da sua contribuição, quem realiza sente prazer e gosta do que faz. }\end{array}$ & \multirow{5}{*}{$\begin{array}{l}\text { Dimensão } \\
\text { Individual }\end{array}$} & \multirow{9}{*}{$\begin{array}{lr}\text { Os sentidos } & \text { do } \\
\text { trabalho } & \text { dos } \\
\text { docentes de PPG } \\
\text { stricto sensu } & \text { das } \\
\text { áreas } & \text { de } \\
\text { Administração } & \text { e } \\
\text { Educação } & \text { do } \\
\text { Estado no } & \text { Rio } \\
\text { Grande do Sul } & \end{array}$} \\
\hline $\begin{array}{l}\text { Sustento - Remuneração, qualidade de vida e realização de desejos } \\
\text { materiais. }\end{array}$ & & \\
\hline $\begin{array}{l}\text { Identidade - identidade pessoal e organizacional, a instituição é reconhecida } \\
\text { e fornece certo status. }\end{array}$ & & \\
\hline $\begin{array}{l}\text { Coerência - quem realiza acredita no que faz, vai ao encontro dos seus } \\
\text { valores pessoais. }\end{array}$ & & \\
\hline Crescimento e aprendizagem. & & \\
\hline $\begin{array}{l}\text { Organização do Trabalho - Não é rotineiro e apresenta novidade, é } \\
\text { considerado útil, tem objetivos claros e resultados, permite autonomia de } \\
\text { pensamento e criação, tem horários e atividades flexíveis. }\end{array}$ & \multirow{2}{*}{$\begin{array}{l}\text { Dimensão } \\
\text { Organizacional }\end{array}$} & \\
\hline $\begin{array}{lll}\text { Condições de Trabalho - Espaço físico adequado, possibilita } & 0 \\
\text { desenvolvimento de relações interpessoais e o reconhecimento. } & \end{array}$ & & \\
\hline Inserção Social & \multirow[b]{2}{*}{ Dimensão Social } & \\
\hline $\begin{array}{l}\text { Contribuição Social - Avanço da área, ético e moralmente aceitável, auxilia } \\
\text { pessoas e contribui para a sociedade. }\end{array}$ & & \\
\hline
\end{tabular}

Figura 2: Categorias da pesquisa

Fonte: Elaborada pelos autores com base nos resultados da pesquisa (2018).

\section{ANÁLISE DOS RESULTADOS}

Os sentidos do trabalho são analisados nas próximas seções a partir das dimensões individual, social e organizacional, para os docentes de PPG stricto sensu, de acordo com a classificação apresentada por Morin et al. $(2003,2007)$.

\subsection{Dimensão Individual}

A dimensão individual do sentido do trabalho aborda os fatores relacionados ao sentido que 0 trabalho assume para a própria pessoa (Morin et al., 2003, 2007). Nesta dimensão, os docentes apontam que um trabalho que tem sentido é fonte de satisfação pessoal. Tanto a satisfação com a vida quanto a satisfação com o trabalho realizado podem depender mais de como o indivíduo vê seu trabalho do que de renda ou prestígio social (Wrzesniewski, McCauley, Rozin, \& Schwartz, 1997). Sendo assim, é um trabalho que proporciona realização pessoal e quem o realiza gosta do que faz, pois o "sentido significa trabalho que dá prazer, um trabalho que dá satisfação" (E07, Priv 2, PPGA). Nesse caso, muitos docentes consideram um trabalho sem sentido "aquele que tu tens que fazer por obrigação de ganhar dinheiro, que tu não gostas" (E11, Publi, PPGA). Por mais que a 
aquisição de bens e serviços necessários à sobrevivência seja um elemento crucial do trabalho (Drenth, 1991), ele é considerado sem sentido quando realizado somente pela remuneração ou quando quem o realiza não gosta do que faz, não se sente satisfeito e realizado, sendo um trabalho que não traz motivações.

No entanto, a remuneração é considerada importante pelos docentes. $O$ trabalho é "um meio de prover para a nossa vida os recursos necessários para termos uma existência digna" (E17, Priv 1, PPGEdu). É um meio de sustentar a si próprio e as pessoas que dele dependem, garantindo autonomia e independência (D'acri, 2003; Lima, Tavares, Brito, \& Cappelle, 2013; Morin, 2001, 2002; Morin et al., 2003, 2007; Oliveira et al., 2004; Piccinini et al., 2005; Prates et al., 2014). A questão da remuneração foi um ponto mencionado por todos os entrevistados, sendo sempre relacionada ao prazer e à satisfação pessoal, pois é "uma forma de sustentar aquilo que eu gosto de fazer, e em algumas atividades do trabalho, o prazer que ele me dá" (E3, Priv 1, PPGEdu). Para os docentes, a remuneração vai além de suprir as necessidades básicas; ela também está relacionada com a qualidade de vida e com a possibilidade de realizações materiais.

Um trabalho que tem sentido está relacionado à contribuição percebida por quem o realiza e aos desafios e às responsabilidades que apresenta. Para os docentes, um trabalho que tem sentido é aquele em que "tu tens uma contribuição para dar a partir também da tua experiência e da tua formação" (E21, Publi, PPGEdu). Para o E24 (Publi, PPGA), o trabalho que tem sentido apresenta desafios e responsabilidades, pois é "uma maneira de eu me testar e de mostrar para mim mesmo que eu consigo fazer as coisas. E de que eu consigo ir além, consigo fazer e atingir". São resultados semelhantes aos obtidos nos estudos realizados por Lima et al. (2013), Morin (2001, 2002, 2004), Morin et al. (2003, 2007), Oliveira et al. (2004), Piccinini et al. (2005), Prates et al. (2014), Paiva, Bendassolli e Torres (2015) e Rodrigues, Barrichello, Igaray, Soares e Morin (2017).

Os docentes consideram o trabalho algo estruturante, pois "é uma forma que eu tenho de me edificar e me construir como pessoa" (E1, Priv 1, PPGEdu), visto que o trabalho os constitui como seres humanos e indivíduos. Como relata o E27 (Priv 1, PPGEdua), a identidade e a constituição de si também estão ligadas à profissão. Dessa forma, os docentes entendem a sua "identidade muito ligada ao fato de eu ser professor e pesquisador. Isso me define em diversas instâncias e em diversos grupos, em sociabilidades distintas. Eu sempre carrego um pouco isso, de ser professor e de ser pesquisador".

Os docentes encontram também fonte de identificação e de status na IES em que trabalham, como relata o E3 (Priv 1, PPGEdu): 'quando eu comecei a ser reconhecido como 'o' E3 da Priv 1, significa que de fato, tu tens uma identificação da instituição, ou com o trabalho que você faz. Muito mais com a instituição do que com 0 trabalho que eu faço". Isso mostra que a IES em que os docentes trabalham contribui significativamente para a sua identidade, funcionando de certa forma como um sobrenome (Morin et al., 2003).

As possibilidades de crescimento pessoal e profissional, assim como 0 aprendizado e o desenvolvimento de competências, são aspectos presentes nas respostas dos entrevistados (Lima et al., 2013; Morin, 2001, 2002, 2004; Morin et al., 2003, 2007; Oliveira et al., 2004; Piccinini et al., 2005; Prates et al., 2014; Paiva et al., 2015; Rodrigues et al., 2017). Para eles, o trabalho é fonte de aprendizado e renovação, para o qual se está sempre estudando e aprendendo com os pares e com os alunos. Para a E2 (Priv 1, PPGA), o trabalho é "uma atividade onde você é obrigado, com prazer, a estar estudando sempre. Isso é uma coisa que me fascina sabe".

Para os entrevistados, também é importante que o trabalho seja coerente com seus valores pessoais. De acordo com a E26 (Publi, PPGA), um trabalho que tem sentido é um trabalho "com o qual eu posso colocar em ação os valores em que eu acredito". Para tanto, o sentido do trabalho para os docentes também parte de acreditar no trabalho que realizam, acreditar que o trabalho desenvolvido tem um papel importante: "eu acredito muito que a educação, ela tem uma força libertadora, construtiva, edificante muito grande" (E1, Priv 1, PPGEdu).

Dessa forma, um trabalho que faz sentido para os docentes, na dimensão individual, proporciona satisfação pessoal, remuneração e qualidade de vida, identidade pessoal e organizacional e oportunidades de crescimento e aprendizagem (Morin, 2001, 2002; Morin et al., 2003, 2007; D'acri, 2003; Prates et al., 2014; Silva \& Braga, 2014; Nascimento et al., 2016). Para os docentes, um trabalho que tem sentido também vai ao encontro dos seus valores pessoais e quem realiza o trabalho acredita na proposta apresentada, acredita no que faz e que isso tem um papel importante (Oliveira et al., 2004; Piccinini et al., 2005).

\subsection{Dimensão Organizacional}


Na dimensão organizacional, segundo Morin et al. (2003, 2007), os indivíduos atribuem sentido ao seu trabalho em função da sua utilidade, propósito, conhecimento do processo produtivo, relacionamento interpessoal e reconhecimento. Os temas mais recorrentes nesta dimensão estão relacionados à organização do trabalho. Para a E21 (Publi, PPGEdu), um trabalho que tem sentido é organizado de forma que não se torne rotineiro, pois "nessa área não existe monotonia, a gente está convivendo com pessoas, as ideias vêm borbulhando", é uma profissão que possibilita renovação e contato com novas perspectivas e ideias, permitindo a renovação do próprio profissional. Os profissionais que possuem um sentido claro do seu papel, da sua utilidade trabalham em favor de seus propósitos, alimentam o autoentendimento, compreendem os propósitos da organização e buscam entender como podem trazer para as organizações as mudanças que valorizam (Steger \& Dik, 2009). Assim, a percepção de que o trabalho realizado é útil, que tenha objetivos claros e que gere resultados também é um fator que dá sentido ao trabalho.

De acordo com a E11 (Publi, PPGA), o trabalho tem sentido se é útil, pois "aquilo que tu fazes tem uma utilidade, mesmo uma utilidade mais intangível, que eu acho que é o trabalho dos professores muitas vezes. Mas tem utilidade, tem importância, move algumas coisas". O trabalho, para ter sentido, também precisa apresentar "o esforço que se faz e o resultado que se tem. Então, no contexto de sala de aula é bem motivante. E também no mundo da pesquisa você ver o teu trabalho de pesquisa sendo publicado em um journal de alto impacto internacional" (E12, Publi, PPGA).

Já a não obtenção de resultados e a falta de utilidade foram citados como características de um trabalho sem sentido. Como relata a E6 (Priv 1, PPGA), os resultados e a utilidade do seu trabalho podem ser percebidos por meio do sucesso dos alunos, dos resultados de suas pesquisas, da sua utilidade para a sociedade.

Esse sucesso por exemplo, dos nossos alunos, também é o sucesso da gente, porque a gente fica muito contente quando vê que um aluno é muito bem colocado no mercado de trabalho [...] quando você vê lá, há um resultado positivo de uma pesquisa [...] ele faz sentido porque ele traz um resultado. Todo esforço, todo empenho, de qualificação profissional, de todo 0 trabalho junto ao aluno, ele trouxe um resultado, que é positivo. (E6, Priv 1, PPGA).

Um trabalho faz sentido também quando permite que o trabalhador tenha autonomia, que possa pensar sobre o seu trabalho e exercer sua criatividade, em que o docente é livre para "discutir, debater, onde eu não sou censurada, onde eu posso colocar o que eu penso e onde eu posso me colocar efetivamente no mundo, acho que é isso" (E26, Publi, PPGA). Dessa forma, um trabalho considerado sem sentido é um trabalho burocrático, que não possibilita autonomia de pensamento e de criação "um trabalho mecânico, repetitivo, que eu tivesse que fazer coisas que eu não considero criativas, que eu não considero produtivas, que eu não considero interessantes" (E15, Publi, PPGEdu).

Outro fator considerado muito importante pelos docentes para que o trabalho tenha sentido é a flexibilidade de horários e de organização das atividades. Por outro lado, a flexibilidade é um dos motivos do aumento da jornada de trabalho, em função da falta de rotina, o que acaba gerando problemas de saúde causados pelo estresse. Mesmo assim, o fato de não ter horário fixo é um ponto muito apreciado pelos docentes, pois "eu não tenho horário fixo na Priv 1 , então eu tenho muita liberdade sobre as minhas atividades, e é superpositivo para mim essa sistemática de liberdade em relação ao horário [...] eu trabalho muito em casa, eu trabalho às vezes de madrugada" (E27, Priv 1, PPGEdu).

As condições de trabalho, ambiente agradável e espaço físico adequado também foram pontos lembrados pelos docentes. Além do espaço físico, o relacionamento com os alunos e com os pares é considerado fundamental, assim como ter um bom grupo de trabalho. Segundo o E17 (Priv 1, PPGEdu), são relações que para alguns vão além da IES e que se tornam relações de amizade. "Não consigo entender o meu trabalho em termos de, individualmente, a gente sempre trabalhou com equipes, com grupos de trabalho. Para mim, isso é um fator muito importante" (E17, Priv 1, PPGEdu). Dessa forma, a relação entre o indivíduo, os outros e o próprio trabalho, molda o sentido do trabalho (Wrzesniewski \& Dutton, 2001).

Para a E1 (Priv 1, PPGEdu), além de ter um bom relacionamento interpessoal, para um trabalho ter sentido, ele deve ser reconhecido, tanto pelos alunos quanto pelos seus pares. "Eu acho que o trabalho com sentido, ele precisa do reconhecimento, essa é uma outra condição, não só dialogo, mas também o reconhecimento" (E1, Priv 1, PPGEdu). Já o isolamento, a precarização e a falta de reconhecimento fazem parte

Revista Alcance - Eletrônica - vol. 25 - n. 3 - Set./Dez. 2018 
das características citadas pelos docentes de um trabalho sem sentido. Assim, um trabalho sem sentido "isola as pessoas também, né" (E1, Priv 1, PPGEdu).

Um trabalho que faz sentido para os docentes, na dimensão organizacional, é um trabalho considerado útil, com objetivos claros, em que se conhecem o seu propósito, os seus processos e os seus resultados, que oportuniza o relacionamento interpessoal e o reconhecimento (Morin, 2001, 2002; Morin et al., 2003, 2007; D'acri, 2003; Oliveira et al., 2004; Piccinini et al., 2005; Coutinho et al., 2011; Menezes et al., 2011; Lima et al., 2013; Paiva et al., 2015; Vilas Boas \& Morin, 2016). 0 trabalho com sentido é organizado de forma que seja diversificado, que não seja rotineiro, que apresente novidades, que permita autonomia de pensamento e de criação (Morin, 2001, 2002; Oliveira et al., 2004; Piccinini et al., 2005). É um trabalho que possui as condições necessárias para sua execução e que possui flexibilidade em seus horários e arranjos das atividades. Sendo assim, um trabalho com sentido engaja as pessoas com características desejáveis dentro das organizações, apresentando menor risco de rotatividade, maior comprometimento e envolvimento com as atividades desempenhadas (Steger et al., 2012).

\subsection{Dimensão Social}

Para os docentes, o trabalho que faz sentido é visto como um trabalho que contribui para a sociedade, que contribui e ajuda a melhorar o mundo, a transformar as pessoas. Segundo a E21 (Publi, PPGEdu), a contribuição social também passa pelo avanço da área, a partir das pesquisas realizadas e das publicações, pois "avançar o conhecimento na área que a gente atua, é transformar. Por isso que a gente sempre diz, é o estado da arte. A gente precisa conhecer, mas também, a partir daquilo, o que a gente pode propor?" (E21, Publi, PPGEdu).

As pesquisas também contribuem para sociedade na medida em que trabalham para resolver problemas sociais, desenvolver novas tecnologias e melhorar a vida das pessoas. Mas a contribuição social relatada pelos docentes como a mais significativa é a transformação na vida dos alunos. 0 crescimento e o desenvolvimento dos alunos se refletem na sociedade, pois os alunos são considerados como multiplicadores de conhecimento, como ressalta o E10 (Publi, PPGA): "Acho que essa é a minha contribuição, tentar juntar essas coisas, fazer com que as pessoas pensem diferente, e os alunos também, pois eles são multiplicadores". Além de multiplicarem esse conhecimento, existe também a preocupação com a formação de pessoas críticas e que possam contribuir para a sociedade, pois "na sala de aula eu não estou formando um empregado, eu estou contribuindo para formar um cidadão brasileiro, um profissional que reflete e que pensa a sociedade, através da sua profissão, é claro, porque está ali no processo de formação de uma profissão" (E26, Publi, PPGA).

Um trabalho com sentido também é digno, que tem um compromisso ético e deve ser exercido com responsabilidade, pois se trabalha com a formação das pessoas. Os alunos veem os docentes como exemplos, acreditam no trabalho dos docentes e no que eles dizem, e cuidar o que diz é considerado pelos entrevistados como um exercício de ética.

Para os docentes, o trabalho é considerado como "a forma como eu vejo o mundo hoje, a minha forma de produção, a minha forma de inserção no mundo" (E9, Publi, PPGA). Assim, o trabalho que tem sentido também proporciona inserção social. É por meio do trabalho que se pode interagir com pessoas além do círculo familiar, se manter atualizado, se relacionar, ter um papel, um lugar no mundo, sendo assim, considerado um fator de sociabilidade.

Dessa forma, para que o trabalho tenha sentido, ele deve contribuir para a sociedade, ser moralmente aceitável e proporcionar inserção social (Morin et al., 2003; Oliveira et al., 2004; Piccini et al., 2005; Lima et al., 2013; Rodrigues, Barrichello, \& Morin, 2016; Rodrigues et al., 2017). Para os docentes, um trabalho que contribui para a sociedade é um trabalho que contribui para o avanço científico da área em que atuam, que auxilia na formação e na transformação das pessoas e que contribui para uma mudança social. A inserção social se dá por meio do relacionamento interpessoal, pelas condições que a profissão possibilita de interagir com pessoas no mundo e pelo sentimento de pertencimento ao mundo, de ter um papel na sociedade que ajude a construir um mundo melhor.

\subsection{Semelhanças e Diferenças do Sentido do Trabalho para os Docentes de Pós-Graduação Stricto Sensu das Áreas de Administração e Educação}


A partir das entrevistas realizadas foi possível determinar as características de um trabalho que tem sentido para os docentes de PPG stricto sensu. Para os docentes de ambas as áreas, um trabalho que tem sentido é organizado de forma que a execução das tarefas permita o exercício de suas competências, que possibilite 0 aprendizado e o crescimento, que seja possível atualizar-se e desenvolver novas competências. É um trabalho que apresenta desafios a serem superados e que ao mesmo tempo vai ao encontro de seus valores e interesses, podendo perseguir seus ideais.

As diferenças encontradas referem-se à organização do trabalho docente. Essas diferenças estão relacionadas ao tipo de IES e são apresentadas questões como a falta de recursos financeiros, enxugamento de pessoal, problemas de infraestrutura e apoio relatados pelos docentes da IES Publi. Com relação às diferenças entre as áreas, o que foi encontrado é que os docentes da área de Administração lecionam mais em PPG lato sensu. Dos dezoito docentes entrevistados da área de Administração, nove lecionam em cursos lato sensu, enquanto que, dos nove docentes entrevistados da área da Educação, apenas um afirmou que ministra aulas nesta modalidade.

Também foi possível perceber que existem diferenças de opiniões dentro da área de Administração relacionadas às métricas de avaliação da CAPES. Para os docentes de áreas mais relacionadas à tecnologia, às engenharias, à sustentabilidade e à produção, as métricas são adequadas e servem como fator motivacional. Enquanto que, para os docentes das áreas mais relacionadas à gestão de pessoas e estudos organizacionais, as métricas são inadequadas, pois uniformizam as especificidades das áreas, o que acaba gerando problemas. Isso se deve às diferenças nas produções, nas oportunidades de publicações e de inserção internacional.

\section{CONSIDERAÇÕES FINAIS}

É por meio do trabalho que o homem modifica o mundo e a si mesmo. O homem se constrói assim, agindo sobre a natureza. E a partir do momento que ele age sobre a natureza ele se transforma: essa é a essência do que é trabalho. Mesmo com todas as transformações que o mundo do trabalho passou, é importante que o trabalho tenha sentido para quem o realiza, sendo assim uma atividade que desenvolve, enriquece e abre caminhos para a autorrealização (Drenth, 1991). Para tanto, o presente estudo buscou analisar os sentidos do trabalho para docentes de PPG stricto sensu das áreas de Administração e Educação.

Assim, para os docentes entrevistados, o trabalho possui sentido de vinculação e pertencimento, que contribui para a sociedade e se recebe remuneração para realizá-lo, o que vai ao encontro da pesquisa realizada pelo grupo MOW (1987). A remuneração também foi algo relacionado ao trabalho em todos os relatos, sendo considerado um ponto importante, assim como os resultados encontrados nas pesquisas realizadas pelo grupo MOW (1987), Morin (2001, 2002), D'Acri (2003), Morin et al. (2003, 2007), Lima et al. (2013) e Prates et al. (2014).

$\mathrm{Na}$ dimensão individual, um trabalho que tem sentido para os docentes é aquele que proporciona satisfação pessoal e quem o realiza sente prazer e gosta do que faz, estando relacionado à ideia de realização pessoal. É um trabalho que possui desafios, responsabilidades e que é considerado importante por quem realiza. Fornece identidade, sendo que a própria identidade dos docentes se confunde com o seu trabalho, estando também relacionada com a IES onde trabalham, sendo considerada fonte de orgulho e reconhecimento social (Morin, 2001, 2002; Morin et al., 2003, 2007; Oliveira et al., 2004; Piccinini et al., 2005).

O sustento e a qualidade de vida também surgem como temas relacionados à dimensão individual, assim como o crescimento e a aprendizagem. As possibilidades de crescimento pessoal e de aprendizagem são aspectos considerados muito importantes, pois o trabalho docente exige qualificação permanente, com a qual se aprende com os pares e com os alunos (Morin, 2001, 2002; Morin et al., 2003, 2007; D'acri, 2003; Oliveira et al., 2004; Piccinini et al., 2005; Prates et al., 2014; Silva \& Braga, 2014; Nascimento et al., 2016). Assim, para os entrevistados é importante que 0 aspecto profissional seja coerente com seus valores pessoais. Como apontam Oliveira et al. (2004) e Piccinini et al. (2005), um trabalho que tem sentido é coerente com os valores e as crenças de quem o realiza. Do contrário, pode se tornar fonte de sofrimento, deixando de ter sentido.

Na dimensão organizacional, um trabalho que tem sentido é organizado de forma que não se torne rotineiro, que seja útil, que tenha objetivos claros e que gere resultados. É um trabalho que permite autonomia de pensamento e criatividade, em que 0 docente possa pensar suas atividades. A flexibilidade de horários e de organização das atividades também é um fator considerado importante, assim como as condições de trabalho, os relacionamentos interpessoais e o reconhecimento por parte dos pares e alunos (Morin, 2001, 2002; Morin et al., 
2003, 2007; D'acri, 2003; Oliveira et al., 2004; Piccinini et al., 2005; Coutinho et al., 2011; Menezes et al., 2011; Lima et al., 2013; Paiva et al., 2015; Vilas Boas \& Morin, 2016).

Esses resultados corroboram os apontamentos realizados por Morin et al., $(2003,2007)$, segundo os quais um trabalho que faz sentido é um trabalho considerado útil, com objetivos claros, em que se conhecem o seu propósito, os seus processos e os seus resultados, que oportuniza o relacionamento interpessoal e 0 reconhecimento (Morin, 2001, 2002; Morin et al., 2003, 2007). Também apresentam semelhanças com os resultados encontrados em estudos realizados por Oliveira et al. (2004) e Piccinini et al. (2005), em que o trabalho que tem sentido é organizado de forma que seja diversificado e não seja rotineiro. É um trabalho que permite autonomia de pensamento e de criação e que possui as condições necessárias para sua execução.

Para os docentes, o trabalho que faz sentido na dimensão social é um trabalho que contribui para a sociedade, que ajuda a melhorar o mundo e a transformar as pessoas. A contribuição social passa pelo avanço da área a partir das pesquisas realizadas e das publicações, as quais também contribuem para sociedade na medida em que buscam resolver problemas sociais, desenvolver novas tecnologias e melhorar a vida das pessoas. 0 crescimento e o desenvolvimento dos alunos e a preocupação com a formação de pessoas críticas, que possam contribuir para a melhoria e a transformação da sociedade, também são características de um trabalho que tem sentido para os docentes, bem como ser um trabalho ético e moralmente aceitável. $O$ trabalho é uma forma de inserção social, por meio do qual se pode interagir com pessoas além do círculo familiar, manter-se atualizado, sendo considerado um fator de sociabilidade (Morin et al., 2003, 2007; Oliveira et al., 2004; Piccinini et al., 2005; Lima et al., 2013; Rodrigues et al., 2016; Rodrigues et al., 2017).

Frente ao exposto, o sentido do trabalho para os docentes de PPG stricto sensu das áreas de Administração e Educação apresentou pouca variedade nas respostas, não apresentando diferenças significativas entre a área de Administração e Educação. As diferenças encontradas estão relacionadas à organização do trabalho docente e ao tipo de IES. Os docentes da IES Publi relataram falta de recursos financeiros, enxugamento de pessoal, problemas de infraestrutura e apoio, enquanto que os docentes das IES Priv 1 e Priv 2 mostraram-se satisfeitos com as condições de trabalho e a infraestrutura oferecidas pelas IES. Outro aspecto é a quantidade de docentes que lecionam em cursos de PPG lato sensu, sendo nove da área de Administração e apenas um da área da Educação.

Cabe ressaltar também a existência de diferentes opiniões em relação às métricas de avaliação dos cursos de PPG stricto sensu realizada pela CAPES entre os docentes da área de Administração. Para alguns docentes das áreas mais relacionadas às engenharias, à produção e à inovação, as métricas são adequadas e servem como fator motivacional. Entretanto, para os docentes das áreas mais relacionadas à gestão de pessoas e estudos organizacionais, as métricas uniformizam as especificidades das áreas, gerando problemas em função das diferenças nas produções e nas oportunidades de publicações e de inserção internacional.

O estudo aqui apresentado contribui para compreender como o trabalho é visto e a sua importância para os indivíduos analisados, assim como o que os leva a encontrar sentido no seu trabalho. 0 estudo apresenta resultados que mesclam os sentidos do trabalho encontrados por Morin $(2001,2002)$ e Morin et al. $(2003,2007)$, aos sentidos do trabalho relatados por Oliveira et al. (2004) e Piccinini et al. (2005) em seus estudos, ampliando assim o quadro que compõe as características de um trabalho que tem sentido. Isso pode estar relacionado ao alto grau de instrução dos entrevistados. Alguns deles, inclusive, pesquisam sobre assuntos relacionados ao mundo do trabalho e sobre os sentidos do trabalho e, por vezes, apresentaram respostas baseadas na literatura sobre o tema.

Como apresentado no estudo, a organização do trabalho está relacionada aos sentidos atribuídos ao trabalho pelos docentes. Assim, os resultados da pesquisa podem auxiliar gerencialmente as IES em questões referentes a uma melhor organização do trabalho docente. Principalmente as questões relatadas pelos docentes relacionadas à burocracia, aos excessos de reuniões, à carga excessiva de trabalho, à má divisão dos cargos de gestão. E no caso das IES públicas, o sucateamento da infraestrutura, o enxugamento de pessoal, a redução de recursos para pesquisa e a falta de apoio para a realização das atividades docentes.

Os resultados encontrados têm pouca variedade nas respostas, não apresentando diferenças significativas entre as áreas de atuação dos docentes, ou quanto ao sexo, à idade ou ao tempo de atuação profissional. Nesse sentido, seria importante ampliar o estudo a outras áreas de atuação dos docentes de PPG stricto sensu, bem como entender o impacto das demandas da gestão da IES com as avaliações da CAPES no trabalho docente. 


\section{REFERÊNCIAS}

Alcadipani, R. (2011). Resistir ao Produtivismo: uma ode à perturbação acadêmica. Cadernos EBAPE.BR, 9(4), 1175-1178.

Antunes, R. (2009). Os Sentidos do Trabalho: ensaio sobre a afirmação e a negação do trabalho (2a ed.). São Paulo: Boitempo.

Bandeira-de-Mello, R. (2010). Softwares em pesquisa qualitativa. In: C. K. Godoi, R. B. Mello, \& A. B. Silva (Org.). Pesquisa qualitativa em estudos organizacionais (2a ed.). São Paulo: Saraiva.

Bardin, L. (2011). Análise de conteúdo (1a ed.). São Paulo: Edições 70.

Bastos, A. V. B., Pinho, A. P. M., \& Costa, C. A. (1995). Significado do trabalho: um estudo entre trabalhadores inseridos em organizações formais. Revista de Administração de Empresas, 35(6), $20-29$.

Bendassolli, P. F. (2009). Psicologia e Trabalho: apropriações e significados. São Paulo: Cengage Learning.

Berg, J. M., Dutton, J. E., \& Wrzesniewski, A. (2013). Job crafting and meaningful work. Purpose and meaning in the workplace, 81, 104.

Bianchetti, L., \& Machado, A. M. N. (2009). Publicar \& Morrer!? Análise do impacto das políticas de pesquisa e pós-graduação na constituição do tempo de trabalho dos investigadores. Educação, Sociedade \& Culturas, (28), 53-69.

Costa, F. J., Moreira, J. A., \& Ethur, S. Z. (2006). O perfil dos professores de pós-graduação em administração na perspectiva dos alunos. Anais do Encontro Nacional da Associação Nacional de Pós-Graduação e Pesquisa em Administração, Rio de Janeiro, Brasil, 30.

Coutinho, M. C., Magro, M. L. P. D., \& Budde, C. (2011). Entre o prazer e o sofrimento: um estudo sobre os sentidos do trabalho para professores universitários. Psicologia: Teoria e Prática, 2(13), 154-167.

D'acri, V. (2003). Trabalho e saúde na indústria têxtil de Amianto. São Paulo em Perspectiva, 2(17), 13-22.

Dejours, C. (2013). A sublimação, entre sofrimento e prazer no trabalho. Revista Portuguesa de Psicanálise, 33(2), 9-28.

Duarte, N. (2006). A pesquisa e a formação de intelectuais críticos na pós-graduação em Educação. Perspectiva, 24(1), 89-110.

Drenth, P. J. D. (1991). Work meanings: a conceptual, semantic and developmental approach. The European Work and Organizational Psychologist, 1(2-3), 125-133.

Forattini, C. D., \& Lucena, C. A. (2015). Adoecimento e sofrimento docente na perspectiva da precarização do trabalho. Laplage em Revista, 1(2), 32-47.

Freitas, M. E. (2007a). A carne e os ossos do ofício acadêmico. Organizações \& Sociedade, 14(42), 187-191.

Freitas, M. E. (2007b). Ser ou estar acadêmico? Organizações \& Sociedade, 14(43), 199-204.

Freitas, M. E. (2011). O pesquisador hoje: entre 0 artesanato intelectual e a produção em série. Cadernos EBAPE.BR, 9(4), 1158-1163.

Godoi, C. K, \& Mattos, P. L. C. L. (2010). Entrevista qualitativa: instrumento de pesquisa e evento dialógico. In: C. K. Godoi, R. B. Mello, \& A. B. Silva (Org.). Pesquisa qualitativa em estudos organizacionais (2a ed.). São Paulo: Saraiva.

Hackman, J. R., \& Oldham, G. R. (1975). Development of the Job Diagnostic Survey. Journal of Applied Psychology, 60(2), 159.

Heloani, R., \& Lancman, S. (2004). Psicodinâmica do trabalho: o método clínico de intervenção e investigação. Revista Produção, 14(3), 77-86.

Karawejczyk, T. C., \& Estivalete, V. de F. B. (2002). O sentido do trabalho e o desenvolvimento de competências: perspectivas sob a ótica do professor universitário. Anais do Encontro de Estudos Organizacionais, Observatório da Realidade Organizacional: PROPAD/UFPE, Recife, Brasil, 2. 
Lima, M. P. de, Tavares, N. V., Brito, M. J., \& Cappelle, M. C. A. (2013). O sentido do trabalho para pessoas com deficiência. Revista de Administração Mackenzie, 14(2), 42-68.

Maués, O. C., \& Mota Jr., W. P. (2011). A nova regulação educacional e o trabalho docente na pós-graduação brasileira. Linhas Críticas, 17(33), 385-402.

Menezes, L. M. de, Napomuceno, L. H., \& Batista-dos-Santos, A. C. (2011). Os sentidos do trabalho para um grupo de professores de uma universidade pública: a dialética prazer-sofrimento em tempos de flexibilidade. Anais do Encontro Nacional de Programas de Pós-Graduação em Administração, Rio de Janeiro, Brasil, 35.

Moreira, M. L., \& Velho, L. (2008). Pós-Graduação no Brasil: da concepção "ofertista linear" para "novos modos de produção do conhecimento" implicações para avaliação. Avaliação, 13(3), 625-645.

Morin, E. M. (2001). Os sentidos do trabalho. Revista de Administração de Empresas, 41(3), 8-19.

Morin, E. M. (2002). Fator humano: os sentidos do trabalho. Revista de Administração de Empresas executivo, 1(1).

Morin, E. M., Tonelli, M. J., \& Pliopas, A. L. V. (2003). O trabalho e seus sentidos. Anais do Encontro Nacional de Programas de Pós-Graduação em Administração, Atibaia, Brasil, 27.

Morin, E. M., Tonelli, M. J., \& Pliopas, A. L. V. (2007). O trabalho e seus sentidos. Psicologia \& Sociedade, 19(1), 47-56.

Mow International Research Team. (1987). The meaning of working. Academic Pr.

Nascimento, R. P., Costa, D. V. F., Salvá, M. N. R., Moura, R. G. de, \& Lutumba, A. S. S. (2016). "Trabalhar é manter-se vivo": envelhecimento e o sentido do trabalho para docentes de ensino superior. Sociedade, Contabilidade e Gestão, 11(2).

Neves, D. R., Nascimento, R.P., Felix Jr, M.S., Da Silva, F.A., \& De Andrade, R.O.B. (2018). Sentido e significado do trabalho: uma análise dos artigos publicados em periódicos associados à Scientific Periodicals Electronic Library. Cadernos EBAPE. BR, 16(2), 318-330.

Oliveira, M. A. M., \& Freitas, M. V. M. T. (2009). O Atual Modelo de Avaliação da CAPES: seus impactos sobre as vidas profissional e pessoal dos docentes de um programa de pós-graduação em letras. Educação em Foco, (13), 29-52.

Oliveira, S. R. de, Piccinini, V. C., Fontoura, D. dos S., \& Schuweig. C. (2004). Buscando o sentido do trabalho. Anais do Encontro Nacional de Programas de Pós-Graduação em Administração, Porto Alegre, Brasil, 28.

Paiva, J. C. M., Bendassolli, P. F., \& Torres, C. C. (2015). Sentidos e significados do trabalho: dos impedimentos às possibilidades do trabalho de pessoas com deficiências. Estudos e Pesquisas em Psicologia, 15(1), 218-239.

Patrus, R., \& Lima, M. C. (2014). A Formação de Professores e de Pesquisadores em Administração: contradições e alternativas. Revista Economia \& Gestão, 14(34).

Piccinini, V. C., Oliveira, S. R. de, Fontoura, D. dos S., \& Schweig, C. (2005). Quando trabalhar faz sentido? Anais do 16 e Conférence de L'AGRH, Paris Dauphine, França.

Pizzio, A., \& Klein, K. (2015). Qualidade de vida no trabalho e adoecimento no cotidiano de docentes do ensino superior. Educação \& Sociedade, 36(131), 493-513.

Prates, C., Silva, N. G. da, \& Piccinini, V. C. (2014). O Sentido do Trabalho Para o Operário: Estudo de Caso em uma Fábrica de Componentes Eletrônicos. Ciências Sociais em Perspectiva, 13(24), 139-160.

Rigon, J., \& Vilela, L. D. (2004). El Significado del Trabajo: Homologación de uma Versión em Portugués-Brasileño del Cuestionario Mow. Anais do Encontro Nacional de Programas de Pós-Graduação em Administração, Porto Alegre, Brasil, 28.

Rodrigues, A. L., Barrichello, A. \& Morin, E. M. (2016). Os sentidos do trabalho para profissionais de enfermagem: um estudo multimétodos. Revista de Administração de Empresas, 56(2), 192-208.

Rodrigues, A. L., Barrichello, A., Irigaray, H. A. R., Soares, D. R., \& Morin, E. M. (2017). O trabalho e seus sentidos: um estudo com peritos criminais da Polícia Federal. Revista de Administração Pública, 51(6), 1058-1084. 
Rosso, B. D., Dekas, K. H., \& Wrzesniewski, A. (2010). On the meaning of work: A theoretical integration and review. Research in organizational behavior, 30, 91-127.

Silva, J. M., \& Braga, J. (2014). Os sentidos do trabalho: uma análise circunscrita ao trabalho dos docentes de duas instituições de ensino superior em salvador. Revista Formadores: Vivências e Estudos, 7(1), 46-59.

Steger, M. F., \& Dik, B. J. (2010). Work as meaning: Individual and organizational benefits of engaging in meaningful work. In P. A. Linley, S. Harrington, \& N. Garcea (Eds.), Oxford library of psychology. Oxford handbook of positive psychology and work (pp. 131-142).

Steger, M. F., Dik, B. J., \& Duffy, R. D. (2012). Measuring meaningful work: The work and meaning inventory (WAMI). Journal of Career Assessment, 20(3), 322-337.

Tolfo, S. da R., \& Piccinini, V. C. (2007). Sentidos e significados do trabalho: explorando conceitos, variáveis e estudos empíricos brasileiros. Psicologia \& Sociedade, 1(19), 38-46.

Tolfo, S. da R., Coutinho, M. C., Almeida, A. R. de, Baasch, D., \& Cugnier, J. S. (2005). Revisitando Abordagens Sobre Sentidos e Significados do Trabalho. Anais do Fórum CRITEOS, Porto Alegre, Brasil.

Triviños, A. N. S. (1987). Introdução à pesquisa em ciências sociais: a pesquisa qualitativa em educação. São Paulo: Atlas.

Vilas Boas, A. A., \& Morin, E. M. (2015). Sentido do Trabalho e Orientação para o Trabalho: Um Estudo em Universidade Públicas de Minas Gerais e do Quebec. Revista GUAL, 8(4), 117-133.

Vilas Boas, A. A., \& Morin, E. M. (2016). Sentido do trabalho e fatores de qualidade de vida no trabalho: a percepção de professores brasileiros e canadenses. Revista Alcance, 23(3), 272-292.

Vilas Boas, A. A., \& Morin, E. M. (2017). Qualidade de vida no trabalho: um modelo sistêmico de análise. Revista Administração em Diálogo, 19(2), 62-90.

Vosgerau, D. S. A. R., Orlando, E. de A, \& Meyer, P. (2017). Produtivismo acadêmico e suas repercussões no desenvolvimento profissional de professores universitários. Educação \& Sociedade, 38(138), 231-247.

Wrzesniewski, A., \& Dutton, J. E. (2001). Crafting a job: Revisioning employees as active crafters of their work. Academy of management review, 26(2), 179-201.

Wrzesniewski, A., McCauley, C., Rozin, P., \& Schwartz, B. (1997). Jobs, careers, and callings: People's relations to their work. Journal of research in personality, 31(1), 21-33. 